\title{
p53 plays a central role in lymphatic anomalies
}

\author{
Rohan Mylavarapu ${ }^{1}$, Molly R. Kulikauskas' ${ }^{1}$, Cathrin Dierkes ${ }^{2}$, Nema Sobhani ${ }^{1}$, Jeffrey Finlon ${ }^{3}$, Michelle Mangette ${ }^{1}$, Wanida Stevens ${ }^{1}$, Farinaz \\ Arbab $^{4}$, Neil F. Box ${ }^{1}$, Mark Lovell5, Ajit Muley ${ }^{6}$, Carrie J. Shawber6, Beth Tamburini ${ }^{3}$, Friedemann Kiefer ${ }^{2}$, Tamara Terzian'1 \\ 1Department of Dermatology, University of Colorado School of Medicine, Aurora, CO, USA \\ 2Max-Planck-Institute for Molecular Biomedicine Department Vascular and European Institute for Molecular Imaging, University of Munster, Munster, Germany \\ 3Division of Gastroenterology, University of Colorado School of Medicine, Aurora, CO, USA \\ 4HCA healthcare, Pathology Department, Tomball, TX, USA \\ ${ }^{5}$ Department of Pathology Children's Hospital Colorado, University of Colorado School of Medicine, Aurora, CO, USA
6Department of Obstetrics and Gynecology, Columbia University Medical Center, New York, NY, USA
}

The lymphatic system plays important roles in draining fluids from interstitial spaces, absorbing lipids from the intestinal tract, and transporting white blood cells to lymphoid organs. Based on these functions, a number of diseases are associated with lymphatic vascular abnormalities including lymphedema, inflammatory disorders, and tumor-related lymphangiogenesis. Thus, understanding the mechanisms underlying the development of the lymphatic network can guide treatment of lymphatic diseases. Activation of the transcription factor p53 has been implicated in several developmental syndromes, where p53 is stimulated by cellular stressors like ribosomal imbalance. p53 then triggers important antiproliferative programs like cell-cycle arrest and apoptosis and is therefore maintained at very low physiological levels during embryogenesis. Here, we report for the first time, a critical role of p53 in defects of lymphatic development, opening the way for new therapeutic avenues. We generated two murine models that carry increased p53 activity induced by ribosomal stress concomitant with the loss of its negative regulators. These high-p53 models are embryonic lethal and present with cutaneous hemorrhaging, severe edema, and distended blood-filled lymphatic vessels. Characterization of the lymphatic endothelial vessels at late-gestation showed a reduced proliferation of endothelial cells that coincided with the upregulation of growtharrest marker p21 and a potential decrease in initial lymphatics that absorb the interstitial fluid. We also demonstrated that the lymphatic phenotypes were p53-dependent as genetic deletion of one copy of p53 or pharmacologic inhibition of p53 abolished the cutaneous hemorrhaging, drastically reduced edema, and rescued the embryonic lethality. Importantly, we detected overexpression of p53 exclusively in lymphatic endothelium from diseased human lymphatic abnormalities, supporting the clinical relevance of our findings. Taken together, we discovered that p53 plays a central role in lymphedema predominantly through cell cycle arrest. Our study also indicated that targeting the p53 pathway, a previously unrecognized mechanism in the genesis of lymphatic deficiencies, may offer therapeutic options for incurable lymphatic maladies.

\section{Main}

The transcription factor p53 is a major sensor of cellular stress, including ribosomal imbalance, DNA damage and oncogene activation $^{1,2}$. Once induced, p53 triggers multiple important cellular programs such as cell cycle arrest, apoptosis and senescence that are deleterious to healthy normal cells ${ }^{3-5}$. Therefore, p53 is tightly regulated by its main inhibitors, Mdm2 and $\mathrm{Mdm}$, and is maintained at undetectable levels in normal fetal and adult cells. Indeed, homozygous deletion of Mdm2 or Mdm4 in mice causes embryonic lethality due to excessive p53 activity resulting in apoptosis or cell cycle arrest respectively6-8. In contrast, Mdm2 or Mdm4 haploinsufficient mice survive to adulthood and reproduce normally, despite an endogenously active $p 53$, unless challenged by cellular stressors like ionizing radiation or oncogene activation. These mice exhibit radiosensitivity, decreased in-vitro transformation potential, and reduced in vivo tumorigenesis ${ }^{9,10}$. Several studies also revealed a role for p53 overexpression in developmental disorders ${ }^{11,12}$. For example, the ribosomopathy model with reduced expression of ribosomal protein $R p / 27 a$, displays endogenously elevated p53 and phenocopies other mouse models expressing high $p 53^{13}$. To examine for a potential genetic interaction between Rpl27a, Mdm2 and Mdm4, and to study the effects of ribosomal stress induced p53 during embryonic development, we created compound mice with low Rpl27a and heterozygosity for Mdm2 or Mdm4 (Rpl27a:Mdm2+/- and Rpl27a:Mdm4+- mice). These mice demonstrated cutaneous hemorrhaging, severe edema and late-gestational lethality. Histopathology examination revealed lymphatic specific defects in both mouse models.

During embryogenesis, the earliest lymphatic endothelial cells arise from the venous branch of the previously established primitive blood vascular network. The lymphatic network in the adult is essential for regulating tissue fluid balance, immune function, and lipid uptake from the gut ${ }^{14}$. Currently, lymphatic function defects have been linked to several pathologies such as obesity, cancer, lymphedema, and inflammation ${ }^{15}$. Lymphedema results from a failure to collect excess tissue fluid from the interstitial space and return it back to the circulation via the thoracic duct. While lymphedema affects $\sim 300$ millions of people worldwide, our understanding of lymphatic development and pathogenesis trails behind that of the blood vascular system. Therefore, current treatments remain largely palliative, including manual lymph drainage and compression garments (Complex Decongestive Therapy) to reduce swelling and drain excessive fluid $^{16}$ and antibiotics to treat the life-long recurrent infections that lymphedema patients suffer from.

In the last two decades, genetic studies have identified several molecular players in the development of the lymphatic network ${ }^{17}$. One of these genes, Prospero Homeobox 1 (Prox-1), is important for maintaining lymphatic identity given its role as the master switch of lymphatic differentiation from the veins. When 
bioRxiv preprint doi: https://doi.org/10.1101/2020.03.18.992784; this version posted April 1, 2020. The copyright holder for this preprint (which was not certified by peer review) is the author/funder, who has granted bioRxiv a license to display the preprint in perpetuity. It is made available under aCC-BY-NC-ND 4.0 International license.

Prox-1 is expressed in a subpopulation of blood endothelial cells (BECs) of the cardinal vein (CV) around E9.5, they give rise to two populations of lymphatic endothelial cells (LECs) ${ }^{18-20}$. The first LEC population sprouts from the venous endothelium starting from E10.5-E11.5 to form the primary lymph sac. These cells also express the lymphatic endothelial markers Hyaluronan Receptor-1 (Lyve-1) and Vascular Endothelial Growth Factor Receptor-3 (Vegfr-3), prompting their proliferation and development into the peripheral lymphatic vessels by E14.514. These vessels eventually sprout into the skin ${ }^{21-25}$, after which they undergo remodeling and maturation to make up the lymphatic network of capillaries and collecting vessels ${ }^{26}$. The second subset of Prox-1+ LECs persists in the CV to form the lymphovenous valves (LVV) that prevent the retrograde flow of blood into the lymphatic circulation 27 . Given the polarized expression and stage-dependent function of Prox-1 during development, dysregulation of this gene can lead to abnormalities within the entire lymphatic system, including reduced proliferation of the lymph vessels and failed separation of the lymphatics from the venous system ${ }^{25}$. Accordingly, mice haploinsufficient in Prox-1 exhibit dermal edema by E13.5, smaller lymph sacs, and lack of functional LVVs ${ }^{21,27,28}$. Additionally, mutations in approximately 12 genes have been found in lymphedema cases 29 , many of which were identified in animal models. Genetic mouse models therefore have been powerful in providing insight into the molecular players of lymphedema and lymphatic-related diseases which allowed the establishment of genetic testing (Lymphatic Malformations and Related Disorders Panel by Blueprint Genetics) that supported a more accurate diagnosis and classification of lymphatic defects. Nevertheless, additional research is needed to identify other factors, genes, and mechanisms that may lead to more therapeutically viable options for lymphedema, since in the majority of cases the underlying genetic basis is unknown. Recently, anti-inflammatory drugs ${ }^{30,31}$ have been effective in managing symptomatic lymphedema that remains with no medicinal option. Our data substantiate that when the master tissue surveyor $\mathrm{p} 53$ goes rogue during development, it can elicit nefarious activities. The lymphatic tissue seems to be particularly sensitive to this p53 gene dosage. Therefore, controlling p53 activity at the stage of lymphatic proliferation may greatly benefit patients with lymphatic disease and relieve them from the ordeals of symptomatic lymphedema.

\section{Results}

Genetic interaction between Rpl27a, Mdm2, and Mdm4 leads to embryonic lethality due to severe cutaneous edema and hemorrhaging

Mice haploinsufficient for $M d m 2, M d m 4$, or Rpl27a displayed an endogenously stable p53 resulting in p53-dependent cellular outcomes such as apoptosis and cell cycle arrest ${ }^{10,13}$. These conditions were subsequently rescued by the deletion of one copy of $p 53$. To test for potential genetic interactions between these genes and observe the impact of an augmented p53 activity, we crossed Rpl27alow/ mice to $M d m 2^{+/-}$or $M d m 4^{+/-}$ animals. From hundreds of crosses, we did not observe the expected double heterozygotes Rpl27alow/+:Mdm2+^ (RP27M2) or Rpl27alow/t:Mdm4+/ (RP27M4). Timed pregnancy (E11.5E18.5) indicated the presence of these genotypes until E16.5 (Tables S1, S2). MRI imaging (Fig. 1a) confirmed these observations and detected the termination of mutant embryos as indicated by the gradual decrease of perfused volume to $0 \mathrm{~mm}^{3}$ between E15.5-E17.5 (Fig. 1b). As we are unable to genotype in utero, we presumed that the deceased fetuses were the double heterozygotes not seen at birth. All the other genotypes increased in total volume proportionally to the gestational age. These data demonstrate an interaction between the three genes that ultimately results in fetal lethality. A closer examination showed that $\mathrm{Mdm}^{+/-}$and $M d m 4^{+/}$embryos were similar to WT with no overt phenotypic abnormalities (SFig. 1). Rp/27alow/t embryos displayed occasional light hemorrhaging and edema starting at E14.5 that was predominantly localized to the dorsal skin (Fig. 1c). These embryos were at a lower body weight and with a developmental delay that persisted until around 8 weeks of age after which they recovered, reproduced and lived normally while slightly underweight ${ }^{13}$. On the other hand, $100 \%$ of RP27M2 and RP27M4 embryos exhibited hemorrhage and/or edema at late-gestation that resulted in $100 \%$ mortality postE16.5 (Fig. 1c). Typically, other mouse models of edema demonstrate lung, heart or liver involvement 32,33 . Examination of Hematoxylin and Eosin (H\&E) staining of major organs such as the heart, lungs, brain or liver of mutant embryos showed no overt abnormalities (SFig. 2a). When we pulled away the bloody skin of both mutants, we did not observe an obvious internal hemorrhaging (SFig. 2b). This led us to check the skin, where we observed large fluid-filled gaps and vessels engorged with blood (Fig. 1d). Hemorrhaging and edema severity scoring on a scale of 0 (none) to 3 (severe) (SFig. 3a) revealed that these conditions gradually worsened with gestational age and ended by death at E16.5. RP27M4 phenotypes were significantly more pronounced than those of RP27M2 (SFigs. 3b, 3c). This is surprising given that Mdm2 is a more powerful inhibitor than Mdm4 due to its E3 ligase activity that degrades p53. Therefore, typically mice with conditional loss of $M d m 2$ in several tissues are invariably much sicker and at an earlier time than those with Mdm4 loss ${ }^{34-36}$

\section{RP27M2 and RP27M4 mice display lymphatic defects}

$R P 27 M 2$ and RP27M4 mutants displayed severe hemorrhaging and cutaneous edema. We performed immunofluorescence staining (IF) of vessels using markers such as Platelet Endothelial Cell Adhesion Molecule-1 (PECAM-1 or CD31) and Lyve-1 respectively. We observed small and flat cutaneous lymph vessels (Lyve-1+) in WT embryos, while RP27M2 and $R P 27 M 4$ lymphatic vessels looked distended and filled with blood (Fig. 2a). The size of lymphatic vessels showed a proportional increase in the severity of the phenotypes. As such, the average vessel diameter for $R P 27 M 4$ measured $\sim 257 \mu \mathrm{m}$, which was approximately two times larger than that of RP27M2 $(\sim 138 \mu \mathrm{m})$, and $\sim 4.3$ times bigger than WT lymphatic vessels $(\sim 58 \mu \mathrm{m})$. Rpl27alow/t lymphatic vessels were also slightly enlarged $(\sim 110 \mu \mathrm{m})$ compared to WT and often filled with erythrocytes (Fig. $2 b$ ). Confocal microscopy on whole-mount embryos stained with lymphatic markers Vegfr-3 and Prox-1 (Fig. 2d) showed free erythrocytes in the interstitial space of the skin and blood-filled (olive color) lymphatics (Vegfr-3+ and Prox$1^{+}$double stained) in the mutants. We recorded sharply reduced density and networking of lymphatics when compared to the other genotypes (Fig. 2c). A change in the distribution of mutant blood vessels (PECAM-1+) was noticed and may be due to pressure exerted from fluid built-up, as no defects in blood vessel formation were detected by whole embryo ultramicroscopy. This was expected as blood vessels develop before lymphatic vessels. The mutants appear to have an increased number of filopodia extended by the LECs and the BECs compared to WT (SFig. 5c), offering the impression that blood and lymphatic vessels are aligned (Supplemental Movies $1,2)$. Given the anti-proliferative role of $p 53$, we double-stained E15.5 skin for Lyve-1 and the proliferation marker Ki-67 or the cell-cycle arrest marker p21. WT, Mdm2+/, Mdm4+- and $R p l 27 a^{l o w /+}$ skin demonstrated an active proliferation in lymphatic 
a

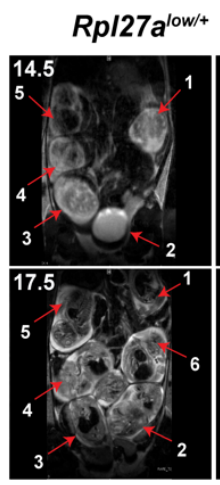

C

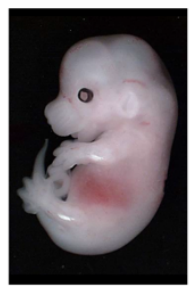

WT

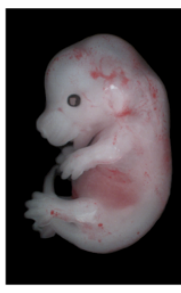

Rp/27a $a^{\text {low/t+ }}$

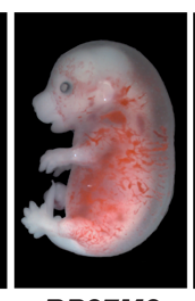

RP27M2
$R p / 27 a^{10 \mathrm{w} / \mathrm{t}} \times \mathrm{Mdm}^{+/-}$

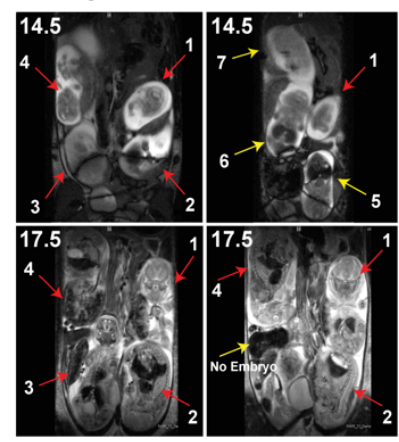

b
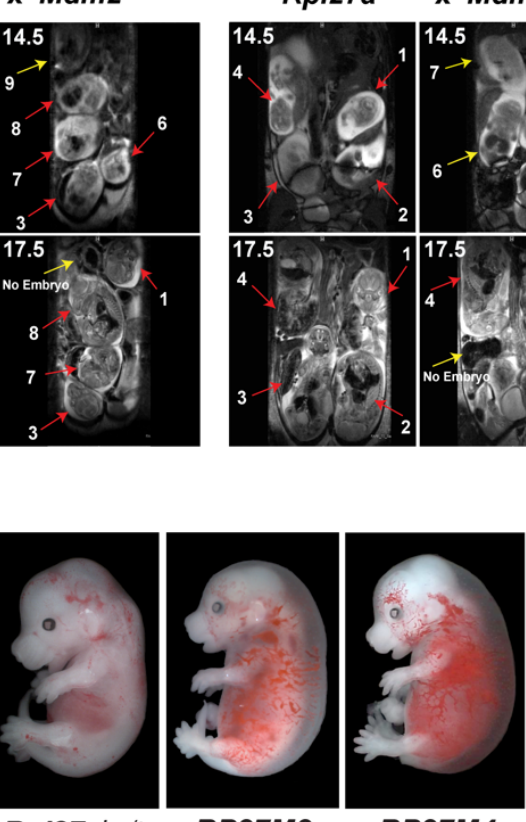

RP27M4

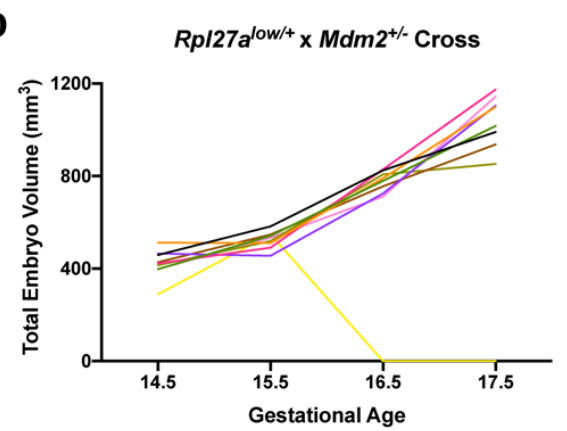

$R p / 27 a^{\text {low/+ }} \times \mathrm{Mdm}^{+/-}$Cross

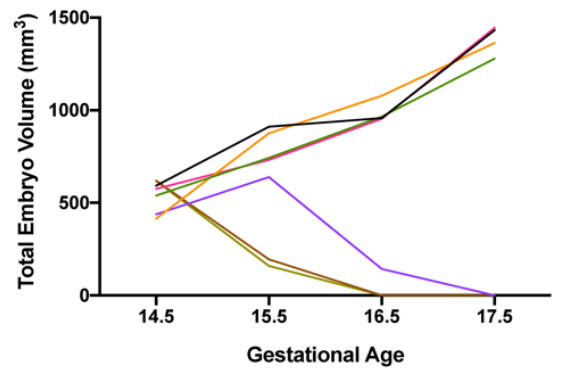

- Embryo 1

- Embryo 2

- Embryo 3

- Embryo 4

- Embryo 5

- Embryo 6

- Embryo 7

d

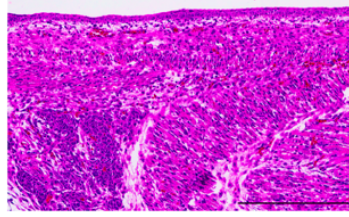

WT

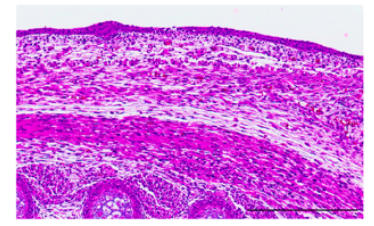

$R p / 27 a^{\text {low/t }}$

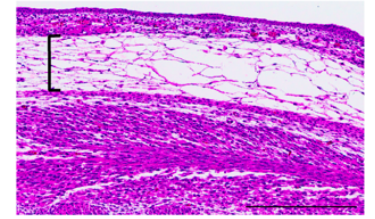

RP27M2

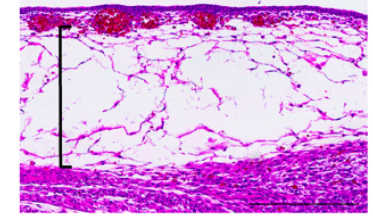

RP27M4

Figure 1. Double heterozygous $R P 27 M 2$ or $R P 27 M 4$ mice are embryonic lethal due to severe edema and cutaneous hemorrhaging. a) MRI scans of $\mathrm{Mdm}^{+/ /}$and $\mathrm{Mdm}^{+/-}$pregnant mice showing coronal sections of fetuses fathered by Rpl27a $\mathrm{a}^{\mathrm{ow} / \mathrm{t}}$ males. Red arrows point to embryos that survived and yellow ones to embryos terminated before E17.5. b) Plot of embryonic volumes as pregnancy progresses. c) Embryonic images at E15.5. d) H\&E staining of E14.5 dorsal skin. Scale bars are $300 \mu \mathrm{m}$. Black brackets show subcutaneous edema.

vessels and an absence of p21 (Figs. 2e, 2f, SFig. 4c, 4d). In contrast, RP27M2 and RP27M4 cutaneous lymphatic vessels showed no detectable Ki-67 and significant upregulation of p21 in mutant LECs (Figs. 2e, 2f). These results indicated a hindrance in proliferation and growth arrest of lymphatic vessels of both models, which may explain the rudimentary network of lymphatics in the mutants. We also double stained for Caspase3 and Prox-1 in four E13.5-E16.5 tissues per genotype to check for apoptosis. Both RP27M2 and RP27M4 embryos showed no obvious upregulation of apoptosis in lymphatic endothelium compared to the other genotypes (data not shown). While we cannot rule out cell death or the presence of senescence, p53 appeared to be acting on the lymphatic network largely through cell-cycle arrest.

Ultramicroscopic imaging of the $\mathrm{CV}$ and its connecting structures at E11.5 showed that the primordial thoracic duct (pTD) and CV were physiologically normal in all embryos. However, the primordial valves, which form the contact side between the PTD and the CV, did not develop properly in RP27M2 and RP27M4 mice (Fig. 3a, SFig.4e). Since several genetic lymphedema models showed lymphatic defects in the mesenteries, we checked E14.5 RP27M2 and RP27M4 mesenteric vessels. Only a few Prox-1+ cells were present at the hilus, but not around the major blood vessels as seen in WT embryos. We also noted very few LECs and reduced lymphatic branching. The small population of LECs present in Rpl27alow/t and mutant mesenteries were rather concentrated near the lymphatic sac, the structure that gives rise to the lymphatic vessels. Prox-1 staining (red) was also detected outside of blood and lymphatic vessels. These Prox-1+ cells were not of lymphatic or blood fate given the absence of Vegfr-3 or PECAM-1 staining respectively (Fig. 3b, SFig. 4f). We speculate that these cells may be macrophages that engulfed Prox-1+ cells but we do not know their exact origin. Staining of E16.5 mesenteries showed that lymphatic vessels were present in Rpl27alow and RP27M2 mice and ran in parallel along the artery-vein pairs that extend from the mesenteric root. In RP27M4, some lymphatic vessels were observed but looked truncated (Fig. 3c, SFig. 4g). These observations indicate that lymphatics eventually develop past E14.5 but with a clear delay to WT and single heterozygous mice.

Gene expression in skin cells of select p53 targets and lymphatic regulators at gestational ages E13.5-E15.5 showed the expected p53 increase in RP27M2 (Fig. 4) and a slight but significant upregulation in Puma ( $p 53$ upregulated modulator of apoptosis) at E14.5 (Fig. 4) that evidently did not translate to 

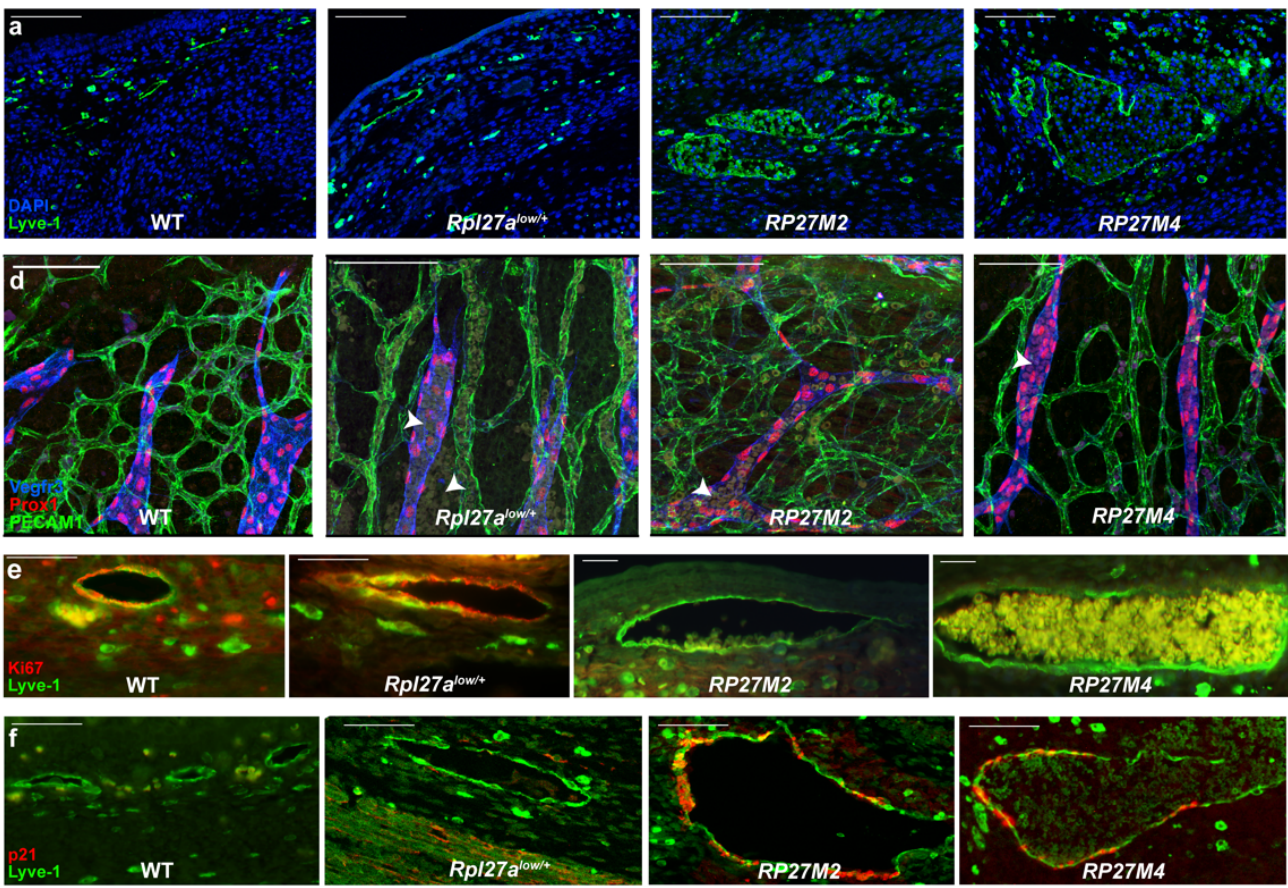
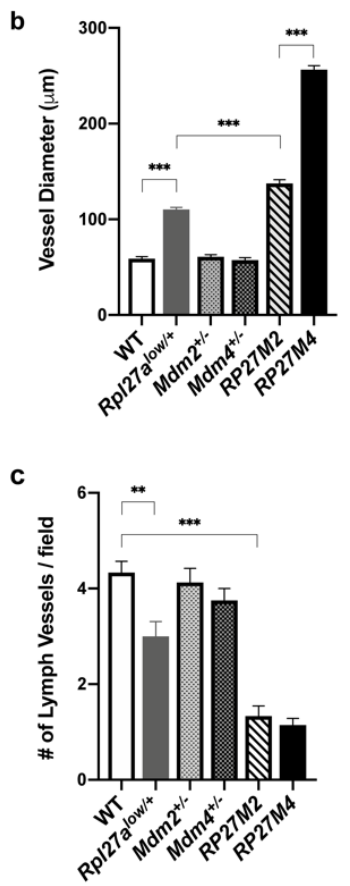

Figure 2. Enlarged E14.5 and E15.5 cutaneous lymphatic endothelium show proliferative defects. a) IF for lymphatic marker Lyve-1 shows distended lymphatic vessels in mutants compared to WT. b) Average lymph vessel size quantification of 10 representative vessels per genotype from $3 \mathrm{WT}, 3 \mathrm{Rpl} 27 \mathrm{a}^{\mathrm{low} / \mathrm{H}}, 2 \mathrm{Mdm} 2^{+/}, 2 \mathrm{Mdm} 4^{+/}, 3 \mathrm{RP} 27 \mathrm{M} 2$, and $3 \mathrm{RP} 27 \mathrm{M} 4$ mice. c) Average number of lymphatic vessels per field from 9 fields at 20X from $3 \mathrm{WT}, 3 \mathrm{Rpl} 27 \mathrm{a}^{\mathrm{low} /+}, 3 \mathrm{Mdm} 2^{+/}$, $3 \mathrm{Mdm} 4^{+/}, 3 \mathrm{RP} 27 \mathrm{M} 2$, and 4 RP27M4 mice. d) Confocal images of whole-mount skin stained for lymphatic markers Vegfr-3 and Prox-1 and general endothelial marker PECAM-1 (CD31) Arrows point to blood (olive color). e) Dorsal embryonic skin double stained with Ki-67 and Lyve-1. Magnification 40X for WT and Rpl27alow/t, 20X for RP27M2 and RP27M4 embryos. f) p21 overexpression in lymphatic endothelium. Data are representative of more than four biological samples per genotype. Scale bars are $100 \mu \mathrm{m}$ for $\mathbf{a}, \mathbf{c}$ and $\mathbf{e}$ and $50 \mu \mathrm{m}$ for $\mathbf{d}$. Statistical significance determined by $t$ test. NS (not significant), ${ }^{\star} p<0.05,{ }^{* *} p<0.01$, and ${ }^{\star \star *} p<0.001$.

increased Caspase-3 positivity in E13.5-16.5 affected tissues (data not shown). Interestingly, p53 overexpression in RP27M4 mice was detected at E13.5 (Fig. 4), which was a day earlier than in RP27M2 mice. This surge of $p 53$ in RP27M4 embryos could partially explain the more severe phenotypes. Puma mRNA was significantly elevated in RP27M4 at E15.5 (Fig. 4). Both RP27M2 and $R P 27 M 4$ skin had dramatically diminished Prox-1 at E15.5 (by $57 \%$ and $73 \%$ respectively) in comparison to $R p / 27 a^{\text {low } /+}$ or WT littermates (Fig. 4), reflecting a potential rarefication of the lymphatic vessels in the skin. Lyve-1 remained elevated at E14.5 and $E 15.5$, ostensibly due to myeloid infiltration as Lyve- 1 is also expressed on macrophages ${ }^{37}$. Vegfr-3, a Prox-1 target whose expression correlates with lymphatic branching, was reduced in both models at E15.5 (Fig. 4). This is consistent with the IF of skin lymphatics that showed fewer vessels in mutant mice (Fig. 2c). Stanczuk et al. (2015) showed that c-Kit+ hemogenic endothelial cells in the mesentery gave rise to lymphatic vessels, indicating that some lymphatics can originate from non-venous hematopoietic progenitors ${ }^{38}$. Since $c-K i t$ is a p53 target that was sharply reduced in the hematopoetic stem cells of Rpl27alow/ mice ${ }^{13}$ and the mesentery of lymphedema models presented by the Mäkinen group, we checked its levels in our mutants. Amazingly, c-Kit was $80-90 \%$ lower in mutant skin compared to WT (Fig. 4), which may contribute to the lymphatic abnormalities in both models. However, we were still perplexed by the accentuated manifestations in RP27M4 versus RP27M2 mice. Since Mdm2 interacts with Mdm4 to regulate $\mathrm{p} 53^{39}$, we checked skin Mdm2 levels. Strikingly, Mdm2 was very low in RP27M4, potentially disrupting the Mdm2-Mdm4 interaction and augmenting p53 activity induced by Mdm2 and Mdm4 haploinsufficiency (Fig. 4). In summary, our findings suggested that p53 upregulation triggered by ribosomal stress led to low Prox-1 and Vegfr-3, impairing normal lymphatic vessel development.

To further characterize the endothelial cell populations in the skin of edemic mice, we separated CD45- stromal cells by FACS sorting using the established endothelial markers PdPn, CD31, and Lyve-140-44 (SFig. 6 and data not shown). We identified four distinct populations: CD31 mid:PdPn low:Lyve-1- (Population I), CD31 high:PdPn low:Lyve-1- (Population II), CD31 mid:PdPn high:Lyve$1^{\text {low }}$ (LECs, Population IIIA) and CD31mid:PdPnhigh:Lyve-1 high (LECs, Population IIIB). No significant differences across these populations were detected in E12.5-E14.5 groups (data not shown). Intriguingly at E15.5, Population / cells drastically accumulated, Population II cells were diminished, and Population III cells were unchanged. A closer look at the state of E15.5 LECs in Population III revealed Lyve-1low (Population IIIA) and Lyve-1 $1^{\text {high }}$ (Population IIIB) subpopulations. Population IIIB was reduced proportionally to the increase in Population IIIA (SFig. 6b). If Population IIIB are the initial lymphatics that absorb the interstitial fluid, their decrease would corroborate the IF stains, where lymphatics were reduced (Fig. 2c) and elucidate the edema in mutants. Thus, it is understandable that the increase in putative collector lymphatics (Population IIIA) did not sufficiently compensate for the drop in initials by merely ramping up the capacity to transport excess fluid back to the vein. 

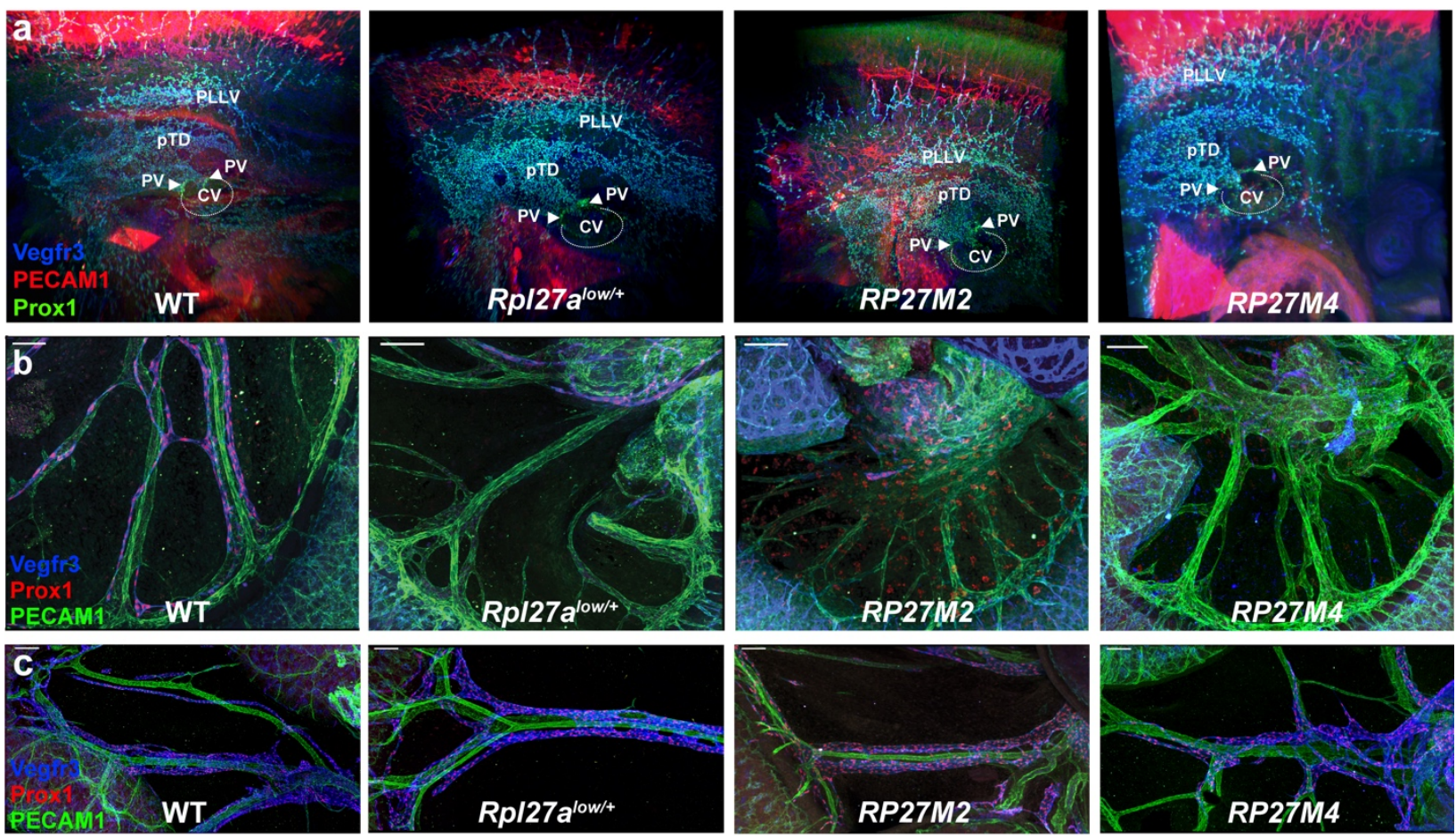

Figure 3. Lymphatic vessels in mutant embryos are less dense or absent but show no obvious defects in early embryogenesis a) Ultramicroscopic imaging of the E11.5 embryo visualizes the primordial thoracic duct (pTD), Peripheral Longitudinal Lymphatic Vessels (PLLV), Primordial Valves (PV), Cardinal Vein (CV), and superficial LECs. b) Confocal images of whole-mount E14.5 mesenteries and c) E16.5 mesenteries. Scale bars are $100 \mu \mathrm{m}$.

\section{p53 haploinsufficiency reversed hemorrhaging, edema and lethality of RP27M2 and RP27M4 embryos}

To determine p53-dependence, we deleted a single allele of $p 53$ in both models. For this, we crossed $R p / 27 a^{l o w /+}$ animals to mice lacking Mdm2 and p53 (Mdm2\%:p53\% mice) or Mdm4 and p53

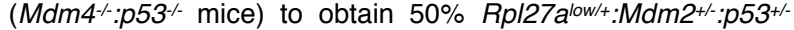

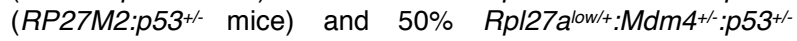
(RP27M4:p53+/) mice respectively. Both crosses resulted in the expected 1:1 Mendelian ratio of progeny (Fig. 5a) that lived to more than 9 months with no edema or hemorrhaging (Figs. 5b,

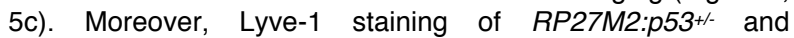
RP27M4:p53+/ skin identified normal lymphatics (Fig. 5c). Molecular examination of RP27M2:p53+/ skin indicated that $p 53$, Puma, and c-Kit returned to WT levels, as did Prox-1, Lyve-1, and PdPn (Fig. 5d, SFig. 6c). Normal gene expression levels were also found in RP27M4:p53+/ skin. Consistent with these data, Population I, II, and III distribution matched the WT (data not shown) and the proportion of Lyve-1+ LECs in Population III was restored after the deletion of $p 53$ (SFig. 6b). Taken together, the aberrant lymphatic development in our mutants was p53dependent, indicating that p53 levels must be kept in check for normal lymphangiogenesis.

Pharmacologic modulation of p53 eliminates cutaneous hemorrhaging, ameliorates edema and rescues embryonic lethality of mutant mice

To investigate p53 as a pharmacologic target for lymphedema, we tested the reversible p53 negative modulator, Pifithrin-a (PFT) ${ }^{45,46}$ in females carrying both models. PFT was injected intraperitoneally (IP) daily from E11.5 to E17.5 and weight gain of females was recorded until delivery. Amazingly, pups from both groups were born at a Mendelian ratio with no signs of hemorrhage (Figs. 6a, 6b), and the females remained healthy. PFT-RP27M2 mice were indistinguishable from WT and 100\% survived until at least 10 days old (Fig. $6 a$ and data not shown). However, PFT-RP27M4 pups had an overall edema that was considerably reduced, slightly looser skin, and excess skin in the area around the neck (Fig. 6a). H\&E staining showed that dorsal skin was normal and without subcutaneous edema in both mutants (Fig. 6b). $100 \%$ of PFT-RP27M4 mice died shortly after birth. Normal size lymphatic vessels were identified by Lyve-1 staining of PFT-RP27M2 and PFT-RP27M4 skin at postnatal day 0 (P0) (Fig. 6c). Since we used a low PFT dose, we speculate that the variability in severity of phenotypes determines the extent of the rescue. Therefore, an increased PFT dose may allow RP27M4 pups to survive. This is a powerful proof of concept that pharmacological modulation of p53 in a critical gestational window is clinically pertinent to treatment of lymphedema where p53 is overexpressed in lymphatic endothelium.

p53 is overexpressed in lymphatic defects and NOT in normal lymphatics

To test for the involvement of p53 overexpression in cases of human lymphatic anomalies, we checked p53 levels by immunostaining in tissues of Lymphatic Malformation associated with severe edema. We observed high p53 positivity in the lymphatic endothelium in six out of eight samples. Interestingly, venous and arterial endothelium were negative in the same p53positive patient tissues (SFig. 7a). Staining for p53 in the endothelial cells of normal neonatal and adult human skin was 
bioRxiv preprint doi: https://doi.org/10.1101/2020.03.18.992784; this version posted April 1, 2020. The copyright holder for this preprint (which was not certified by peer review) is the author/funder, who has granted bioRxiv a license to display the preprint in perpetuity. It is made available under aCC-BY-NC-ND 4.0 International license.
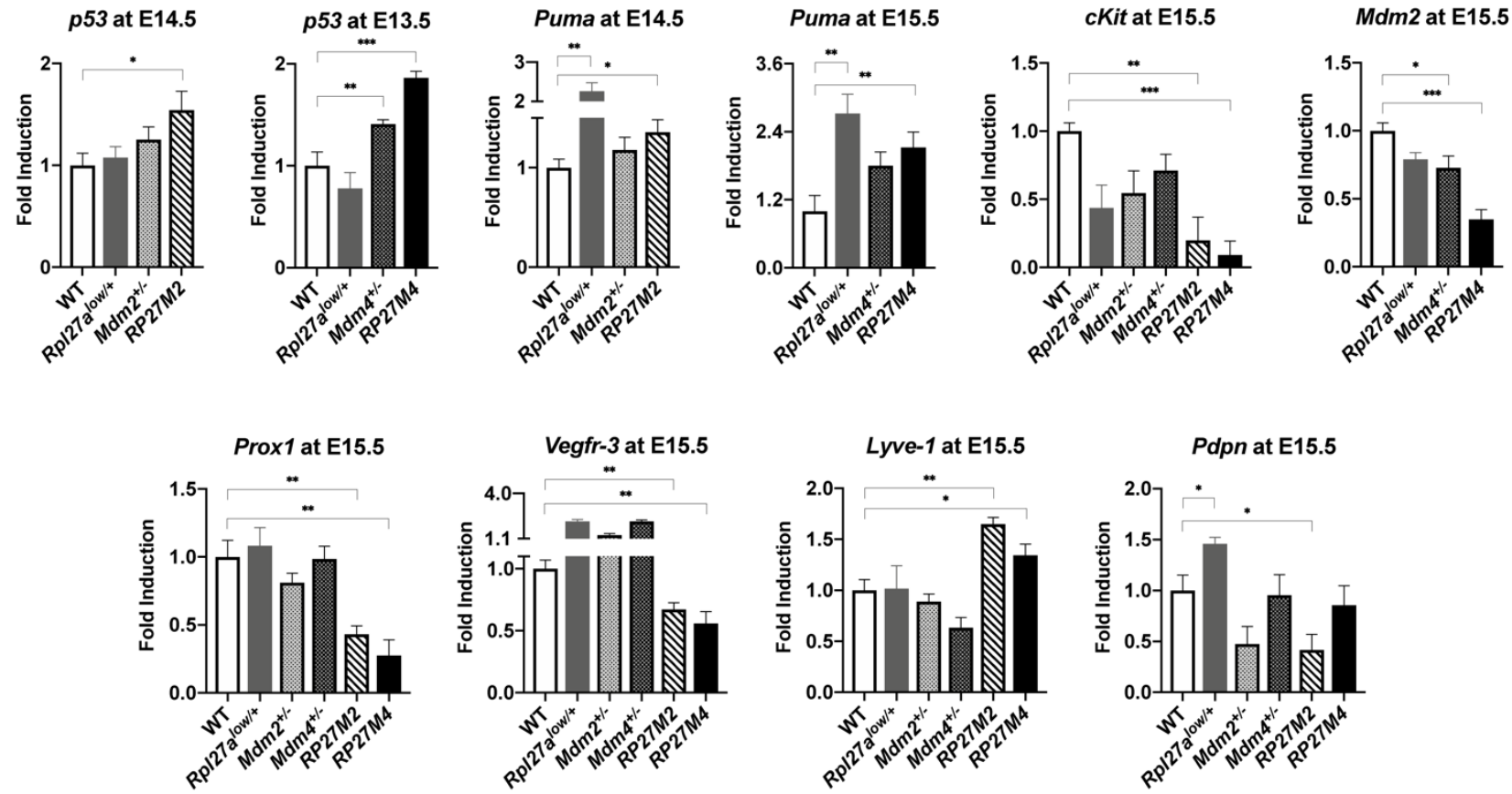

Figure 4. Gene expression assays of RP27M2 and RP27M4 skin show differential expression of p53 targets and lymphatic markers. Gene expression assays (mean + SE) of E13.5 ( $\mathrm{n}=8$ for all genotypes), E14.5 $\left(\mathrm{n}=6\right.$ for all genotypes) and E15.5 skin $\left(\mathrm{n}=7\right.$ for $M d m 4^{+/}$, and $\mathrm{n}=6$ for all other genotypes). Statistical significance was analyzed by $t$ test. NS: not significant, ${ }^{*} p<0.05,{ }^{* *} p<0.01$, and ${ }^{* * *} p<0.001$.

also negative (Figs. 6d, SFig. 7b). In lymphatic diseases, females are more often affected than males (National Organization of Rare Diseases). Therefore, we checked for gender differences in the disease presentation of our mutants. Based on edema and hemorrhage scoring following defined criteria (Fig. S3a), we noted that at E15.5, RP27M2 females were on average two-fold more significantly affected by both hemorrhaging and edema than males. RP27M4 females, however, suffered from more severe hemorrhaging than males but only a tendency for worsening edema (Fig. 6e). Sex differences for edema in RP27M4 mice were likely masked by the extensive magnitude of this phenotype in the mutants. Taken together, p53 upregulation plays a central role in lymphatic defects and our murine models seem like a good representative model of human lymphatic defects.

\section{Discussion}

We show here, for the first time, a link between the transcription factor p53 and the lymphatic system. The characterization of two mouse models of ribosomal stress demonstrated that p53 upregulation leads to lymphedema, establishing the importance of p53 in lymphatic surveillance. A comparative analysis of normal and diseased human lymphatic endothelium identified aberrant p53 levels in lymphatic disorders associated with lymphedema and proposed the p53 pathway as a target for treatment. This is the first report in which pharmacologic modulation of a gene in pre-clinical models of lymphedema reversed lymphatic defects, paving the way for translational research in this cluster of diseases.

p53 upregulation in RP27M2 and RP27M4 mice led to reduced and distended cutaneous lymphatic vasculature filled with blood and a stark delay in the formation of mesenteric lymphatics. These lymphatic phenotypes were first observed at E14.5 (Figs.
$1,2)$, coinciding with the onset of the lymphatic proliferation that establishes the lymphatic network throughout the body. Lymphatic structures continued to develop in the edemic embryos but with a clear delay compared to WT mice, likely due to the considerable drop in Prox-1 and its target Vegfr-3 in these tissues as seen in other lymphedema mice 28,47 (Figs. 3 and 4). Since mouse models of high p53 typically show apoptosis or cellcycle arrest in affected tissues ${ }^{13,34,36,48-50}$, we examined the presence of p53 signatures in our mutants. Despite an overexpression of Puma and Noxa (Fig. 4), no increase in Caspase-3 was detected in RP27M2 and RP27M4 mice (data not shown). While this observation does not preclude a Caspase-independent cell death, the cell cycle arrest due to p21 upregulation (Fig. 2f) points to a clear preferential mode of action of p53 in the lymphatic system. Considering the anti-proliferative function of p53 in tissues, a p21-induced cell cycle arrest in mutant lymphatic endothelium (Fig. $2 \mathrm{~d}$ and $2 \mathrm{e}$ ) was not surprising and resulted in an insufficiency of lymphatic networking and edema in the mutants (Fig. 2d). The phenotypic and overall molecular concordance of both models, as well as the near complete penetrance of their manifestations, strengthened our assumption that WT p53 is the common culprit and asserted that the lymphatic network is particularly sensitive to $p 53$ activity. On the other hand, abnormalities associated with the loss of Mdm4 were accentuated compared to loss of Mdm2 (Figs. 1-3), which is the reverse of what is seen in Mdm2 and Mdm4 gene deletion models $6,7,34-36,48,51 . M d m 2$ is the main p53 inhibitor that, contrary to Mdm4, degrades p53 through its E3 ubiquitin ligase activity ${ }^{39}$, and thus exerts a more powerful control on p53 stability and activity than Mdm4. p53 upregulation in $R P 27 M 4$ a day earlier than in RP27M2 mice (E13.5 skin vs 14.5) might have accentuated the severity of Mdm4-associated lymphedema and hemorrhaging (Fig. 4). Moreover, low Mdm2 levels in RP27M4 skin likely impinged on the cooperation of Mdm4 with Mdm2 to efficiently downregulate p53. Further 
bioRxiv preprint doi: https://doi.org/10.1101/2020.03.18.992784; this version posted April 1, 2020. The copyright holder for this preprint (which was not certified by peer review) is the author/funder, who has granted bioRxiv a license to display the preprint in perpetuity. It is made available under aCC-BY-NC-ND 4.0 International license.

a

\begin{tabular}{|c|c|c|}
\hline Crosses & Genotypes & Progeny observed (expected) * \\
\hline \multirow{2}{*}{ 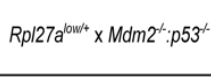 } & $M d m 2^{+/}: p 53^{+/-}$ & $20(22)$ \\
\hline & 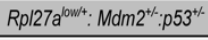 & $24(22)$ \\
\hline \multirow{2}{*}{ 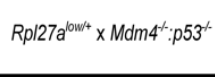 } & $M d m 4^{4^{/ /}}: p 53^{+/}$ & $29(27)$ \\
\hline & 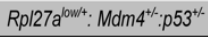 & $25(27)$ \\
\hline
\end{tabular}

b

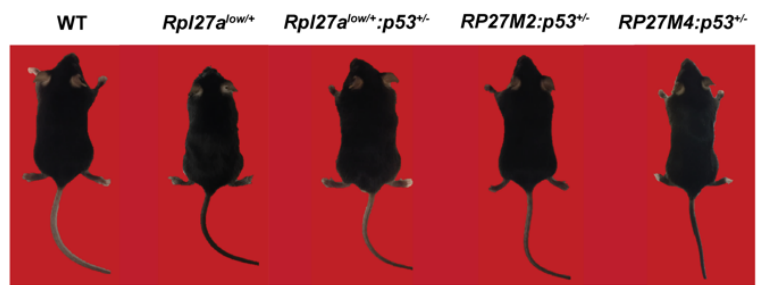

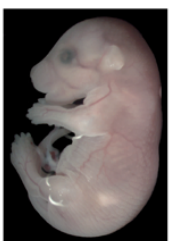

WT

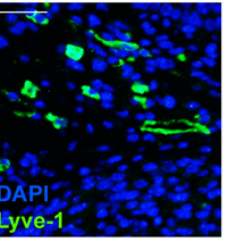

WT

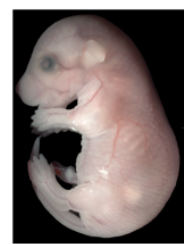

RP27M2:p53

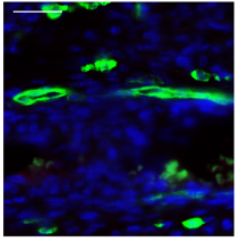

RP27M2:p53 ${ }^{\text {+h }}$

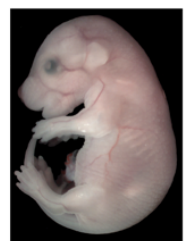

RP27M4:p53 ${ }^{\text {+1- }}$

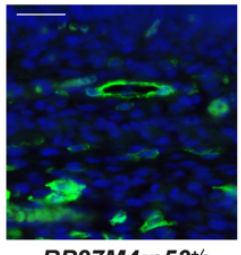

d p53 at E14.5
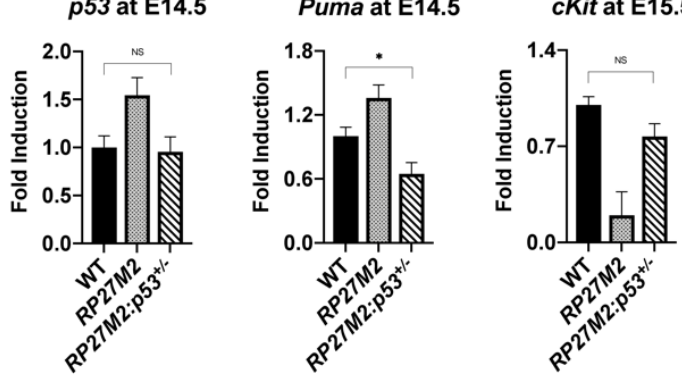

Prox1 at E15.5

Lyve1 at E15.5
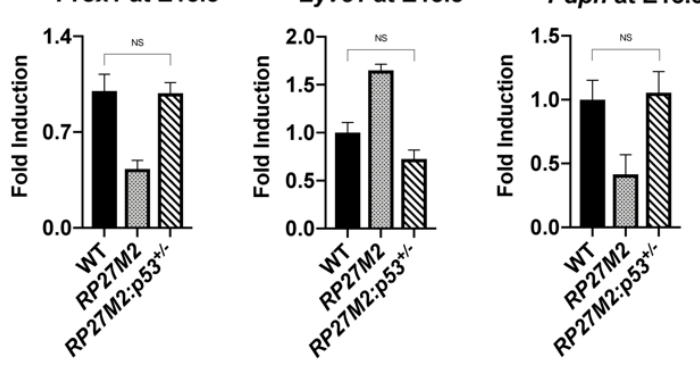

p53 at E13.5

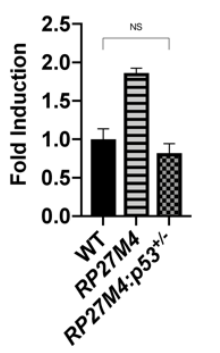

Puma at E15.5

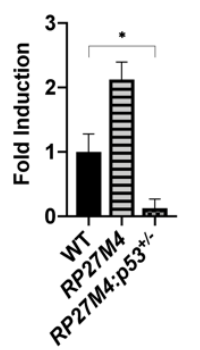

Lyve-1 at E15.5

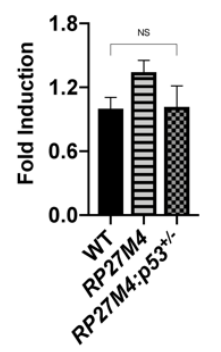

Figure 5. Genetic deletion of one copy of $p 53$ in RP27M2 and RP27M4 mice reverses the lymphatic anomalies and rescues embryonic lethality. a) Progeny of $R p l 27 a^{\text {low } /+}$ mice crossed to $M d m 2^{-}: p 53^{-/}$or $M d m 4^{-}: p 53^{-/}$mice. Mendelian ratio is re-established. b) Representative images of

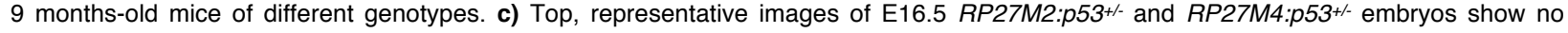
cutaneous hemorrhaging or edema. Bottom, representative images of Lyve-1 immunostaining in E16.5 skin out of 15 imaged vessels per genotype show lymphatic vessels revert to normal size with the deletion of one copy of $p 53$. d) Gene expression assays by qPCR (mean \pm SEM) in RP27M2 and RP27M4 skin with a single allele deletion of $p 53 . \mathrm{N}=8$ for all genotypes at $\mathrm{E} 13.5, \mathrm{~N}=6$ for all genotypes at $\mathrm{E} 14.5$ and $\mathrm{N}=6-7$ at E15.5. ${ }^{*} \mathrm{Chi}$-square test reveals no statistical difference between observed and expected progeny numbers. 20X magnification, scale $100 \mu \mathrm{m}$. Statistical significance determined by $t$ test. NS (not significant), ${ }^{*} p<0.05,{ }^{* *} p<0.01$, and ${ }^{\star * \star} p<0.001$.

investigation into differences between these models can offer unique insights into the specific role of p53 inhibitors in lymphatic vascular development. Nevertheless, both models support a connection between p53 activation and lymphatic defects, largely through impeding proliferation and driving growth arrest (Figs. 2, 3). Another connection to lymphatic disease is the huge decline of $c$-Kit expression in both mutants, reminiscent of other lymphedema models. Stanczuk L. et al. ${ }^{38}$ noted the lymphatic vasculature of the mesentery in mice develop partly from nonvenous c-Kit lineage cells of hemogenic endothelial origin, contrary to the long held doctrine that mammalian lymphatic vessels sprouted from veins. Therefore, $c$-Kit depletion in our mutants conceivably reflects a p53-induced loss of hematopoetic c-Kit+ stem cells that could have contributed to lymphatic anomalies. Of note, high p53 expressing Rpl27alow/t mice also demonstrated some low grade cutaneous hemorrhaging and edema along with less dense lymphatics (Fig. 2c, 3b and SFig. 3b, c). Along this line, mutations in ribosomal protein genes have been highly linked to non-immune hydrops fetalis (NIHF) associated with inherited Diamond-Blackfan Anemia, where a strong role for p53 was demonstrated $52.15 \%$ of NIHF53 have lymphatic abnormalities that could ultimately be explained by a surge in p53. Thus, keeping p53 at bay might restore lymphatic homeostasis; after all, having no p53, as in p53-null mice, does not largely affect gestation ${ }^{8,54}$.

Genetic deletion of one copy of p53 in both RP27M2 and RP27M4 mice reversed symptomatic lymphedema and hemorrhaging, which allowed them to be born at a Mendelian ratio and live normally with no overt pathologies (Fig. 5). In concordance, expression of p53 targets and lymphatic genes in

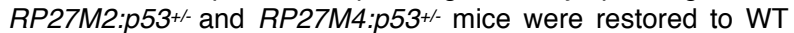
levels (Fig. 5). These findings not only confirmed the p53dependence of lymphatic defects in the mutants but also 
a
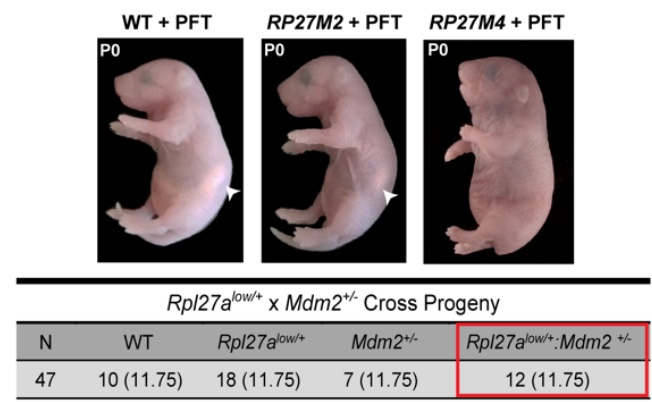

$R p / 27 a^{\text {low/ }} \times M_{M d m} 4^{+/}$Cross Progeny

\begin{tabular}{|cccc|c|}
\hline $\mathrm{N}$ & $\mathrm{WT}$ & Rpl27a $^{\text {low/+ }}$ & $M d m 4^{+/}$ & Rp/27a $^{\text {low/t }}: M d m 4^{+/}$ \\
\hline 40 & $8(10)$ & $10(10)$ & $11(10)$ & $11(10)$ \\
\hline
\end{tabular}

b

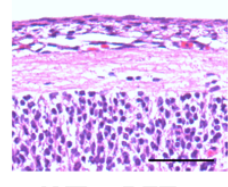

WT + PFT

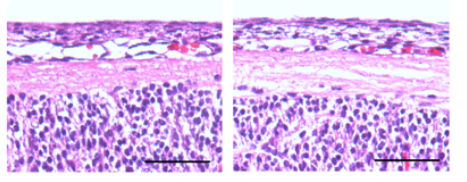

RP27M2 + PFT RP27M4 + PFT
C

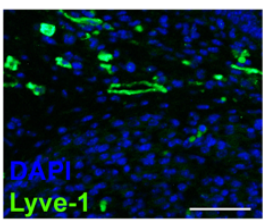

WT + PFT

d

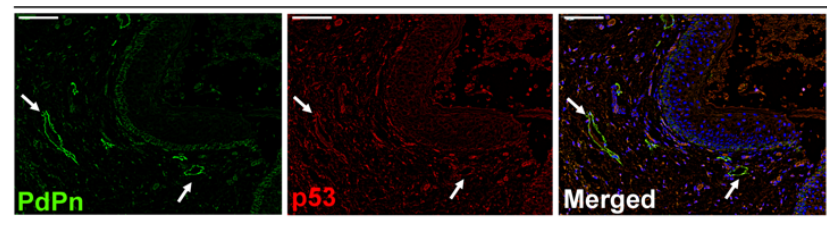

Lymphatic Malformation

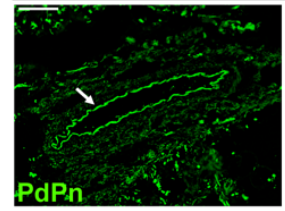

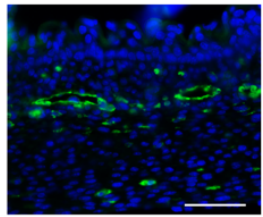

RP27M2 + PFT

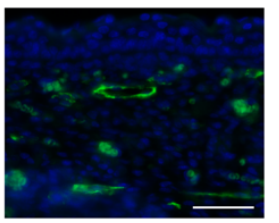

RP27M4 + PFT

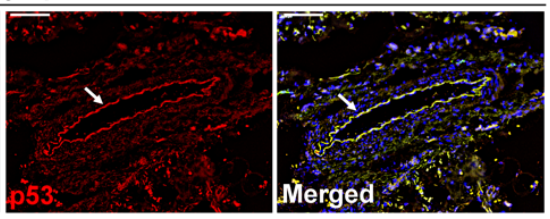

e
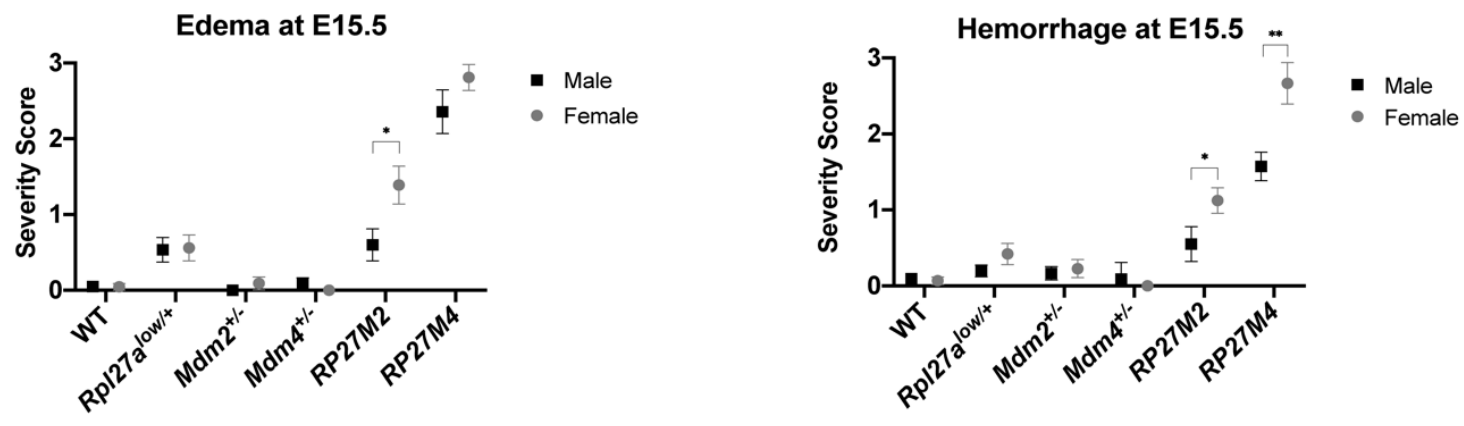

f

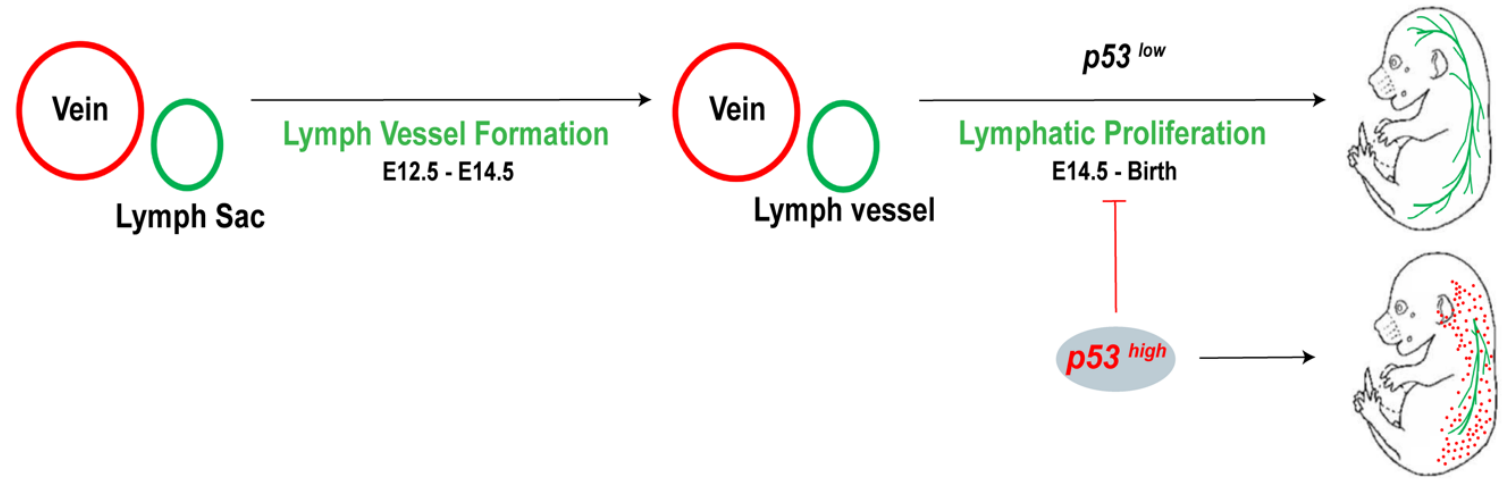

Figure 6. p53 upregulation inhibits lymphangiogenesis. a) Mutant pups born at Mendelian ratio from mothers treated with PFT did not exhibit cutaneous hemorrhaging and edema. Arrows point to milk-spots. b) Representative H\&E of PFT treated P0 and P1 skin. c) Lyve-1 staining of PFT-treated P0 skin. d) PdPn and p53 immunostaining of lymphedema-associated Lymphatic Malformation and neonatal skin control. e) Severity of phenotypes based on sex: WT $(M=14, F=9)$, Rpl27alow/ $(M=28, F=25), M d m 2^{+/}(M=19, F=14), M d m 4^{+/}(M=11, F=8)$, RP27M2 (M = 10, $\mathrm{F}=18$ ), and RP27M4 (M = 7, F = 8). Statistical significance determined by $t$ test. NS (not significant), ${ }^{*} p<0.05,{ }^{* *} p<0.01$, and ${ }^{* \star *} p<0.001$. f) Schematic of proposed mechanism of action of p53 on lymphatic development showing the p53 upregulation inhibits proliferation of lymphatic vessels resulting in insufficiency of lymphatic drainage and cutaneous hemorrhaging. 
bioRxiv preprint doi: https://doi.org/10.1101/2020.03.18.992784; this version posted April 1, 2020. The copyright holder for this preprint (which was not certified by peer review) is the author/funder, who has granted bioRxiv a license to display the preprint in perpetuity. It is made available under aCC-BY-NC-ND 4.0 International license.

proposed p53 as a druggable target in patients with lymphedema-associated disorders. A limitation in this study is that the action of p53 on the lymphatic network could coincidently stem from a non-cell autonomous effect driven by the surrounding cell types. Further investigations will examine the impact of p53 upregulation on LECs due to specifically deleting its negative regulators in this tissue, which may reveal new insights into lymphatic deficiencies. While many studies in genetic models targeted lymphedema, the complexity of the lymphatic network and anomalies has precluded successful drug development. To our surprise, pharmacologic control of p53 levels in both mutants successfully reversed symptoms of edema and hemorrhaging and mice were born following a Mendelian ratio. However, RP27M4 mice were born with loose skin around their neck and did not survive past the first day, perhaps since RP27M4 phenotypes were much more severe than those of RP27M2 mice. Interestingly, the source of dermal lymphatic vasculature in mice varies with location (cervical vs ventral or dorsal) 55,56 , which adds to the complexity of the development and pathology of the lymphatic system. Starting PFT injections of RP27M4 a day early or testing a higher dose of PFT in all likelihood may replicate the results gained from treating RP27M2 mice, unless a p53-independent function of Mdm4 contributes to the severity of lymphatic phenotypes or if p53 does not impact LECs in the neck skin. Nevertheless, attaining a symptomatic reversal in a genetic model of lymphedema through pharmacological treatment, to our knowledge, has not yet been successful. The outcomes with PFT treatment appear, in this context, extraordinary and very promising for translational research ${ }^{31,57}$. Our findings were further corroborated in lymphedema-associated lymphatic disorders. From 8 cases, 6 disease tissue tested positive for p53 while the venous and arterial endothelium from the same patient skin or from normal neonatal and adult samples were negative (Fig. 6 and SFig. 7). This extraordinary finding of drugging the p53 pathway in these anomalies eluded lymphatic research as studies explored causal mutations of lymphatic disease rather than identifying the overexpression/misexpression of WT genes as a cause of mutant phenotypes.

Together, our findings may ultimately lead to novel therapies of lymphedema in the absence of any current pharmacologic avenue. Additionally, understanding the molecular determinants of normal lymphangiogenesis may have a bearing on other related diseases such as obesity, inflammation and cancer 58 . This study, the first to highlight a role of p53 in lymphatic disease, indicates that the lymphatic system is particularly sensitive to p53 gene dosage. Therefore, while p53 is not required for normal formation, p53 needs to remain restricted to circumvent the trigger of its typical anti-proliferative effects that may promote symptomatic lymphedema. We think we know a lot about p53, yet remarkably, we still have much to learn about its functions in tissues, and in particular the lymphatic system. At the time when no medicinal therapy exists to treat lymphedema, p53 may once again come to the rescue.

\section{Acknowledgements}

TT was funded by the NIH/NIAMS K01 AR 063203 award, the University of Colorado School of Medicine Dermatology Department, CCTSI (Child and Maternal Health Program) supported by the NIH/NCATS CTSA Grant UL1 TR002535 and the Dermatology Foundation. NB was supported by the $\mathrm{NIH} / \mathrm{NIAMS}$ R03 AR066880, NIH/NCI R01 CA190533 and the Daneen and Charles Stiefel Investigative Science Award from the American Skin Association. CJS and AM were supported by the DOD grant W81XWH1910266 and NIH/NICHD grant R03
HD092662. Additional funding came from the Cancer Research Summer Fellowship and the Cancer League of Colorado to RM. We thank Dr. Ellen Elias for clinical advice and critical reading of the manuscript, Dr. Chiping Day for his meticulous review of the paper and Dr. Michael Detmar for his input and critical feedback. We would also like to thank Rachel Maxwell and Ellie Mackintosh for technical assistance. We are grateful to Dr. Tracy Lyons and Veronica Wessells for excellent and generous technical assistance with the staining of normal human tissues and advice. Histology of murine histology tissues was prepared by the Cancer Center Histology Core supported by the P30CA046934 grant funding. The MRI was performed by the University of Colorado Animal Imaging Shared Resource (AISR). We thank Dr. Jerrold Ward for detailed histopathological reading of the embryos. We apologize to those whose work we have not cited due to journal citation restrictions.

\section{Materials and Methods}

\section{Mice}

All mice were maintained on a C57BL6/J background. For timed pregnancies, the first day of observed plug were recorded as day 0.5 post-coitum or embryonic day 0.5 (E0.5). Mice haploinsufficient for the ribosomal protein L27a (Rp/27low/+) in the skin ${ }^{13}$ are crossed with $M d m 2^{7}$ or $M d m 4^{34}$ heterozygous mice $\left(\mathrm{Mdm2}^{+/-}\right.$or $\left.\mathrm{Mdm}^{++/}\right)$to generate WT, Rpl27alow/+, $\mathrm{Mdm}^{+/}$, Rpl27alow/+:Mdm2+/- (RP27M2), Mdm4+/, and Rpl27alow/t:Mdm4+/mice (RP27M4). To generate mice on a p53-deficient background, we crossed Rpl27alow/t mice to $M d m 2^{-1}: \mathrm{p5}^{-/}$or

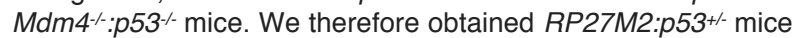
and RP27M4:p53+/- mice. Genotypes were determined by PCR analysis of extracted DNA from tails using published primer sets for Mdm27, Mdm434, and p5359. The sex of embryos was determined by detection of Sry and Raspn genes by PCR 60 . We followed animal care and euthanasia guidelines of the Colorado Institutional Animal Care and Use Committee for all animal work.

\section{Pifithrin-a drug Treatment}

A 10mM stock of Pifithrin-a (PFT- a, Selleck Bio, cat. S2929) was diluted 1:10 in 1X PBS, protected from light and used instantly. Pregnant mice were injected intraperitoneally from $\mathrm{E} 11.5$ to $\mathrm{E} 16.5$ and subcutaneously from E17.5 to the day before delivery at $2.2 \mathrm{mg} / \mathrm{kg}$ of weight. Animal were monitored daily post-treatment and weights were recorded after delivery.

\section{Histology and Immunostaining}

Tissues were fixed in $4 \%$ neutral buffered paraformaldehyde, processed, and embedded in paraffin by the UCD Research Histology core. Sagittal sections $(5 \mu \mathrm{m})$ were subjected to immunofluorescence staining (IF) as previously described ${ }^{13}$ and according to the manufacturer's recommendations. The primary antibodies used for IF were monoclonal mouse Prox-1 (1:50, P21936, Thermofisher Scientific, Massachusetts, USA), monoclonal rabbit Lyve-1 (1:100, ab14917, Abcam Inc., California, USA), polyclonal rabbit Ki-67 (1:1000, VP-K451, Vector Laboratories, California, USA) and mouse monoclonal p21 (1:100, sc-6246, Santa Cruz Biotechnology, California, USA) antibodies. We used anti-rabbit or anti-mouse Alexa Fluor 594 or Alexa Fluor 488 conjugated secondary antibodies (1:1000, Invitrogen, California, USA), captured the images on a Nikon Eclipse 90i, and quantified using the ImageJ software. Pediatric lymphatic edema was categorized using the ISSVA Classification of Vascular Anomalies and confirmed by PdPn (D2-40 antibody, Ventana or RnD Systems AF3670) staining. IHC for p53 on human tissues were performed using the D-07 antibody (Ventana) on automated strainers (Ventana Ultra) following the manufacturer's recommendations and Clinical 
bioRxiv preprint doi: https://doi.org/10.1101/2020.03.18.992784; this version posted April 1, 2020. The copyright holder for this preprint (which was not certified by peer review) is the author/funder, who has granted bioRxiv a license to display the preprint in perpetuity. It is made available under aCC-BY-NC-ND 4.0 International license.

Laboratory Improvement Amendments (CLIA) certified procedures. For the visualization of the three-dimensional lymphatic vasculature, murine skin were fixed in $4 \%$ Paraformaldehyde (PFA) for 2-4 hours at room temperature for whole mount staining. Tissues were then subjected to procedures as published61. H\&E was performed following Harris protocols for staining.

\section{RNA Extraction and quantitative real time PCR}

Mouse embryonic skins were placed in RNA Later (Sigma, cat. R0901) overnight at $4{ }^{\circ} \mathrm{C}$, then stored at $-80^{\circ} \mathrm{C}$ until RNA was extracted. $5 \mathrm{mg}$ of skin was homogenized in 20\% 0.4M DTT in RLT Lysis Buffer (Qiagen, Hilden, Germany). The RNA was isolated using the RNeasy Plus Micro Kit (cat. 73404 and 74004, Qiagen, Hilden, Germany) and their corresponding protocol. Samples with an RNA concentration greater than $500 \mathrm{ng} / \mu \mathrm{L}$ and A280/260 ratio 1.8-2.0 were used for CDNA synthesis. CDNA of $100 \mathrm{ng} / \mu \mathrm{L}$ concentration was synthesized using the SuperScript III First Strand Synthesis SuperMix Kit (cat. 18080-051, ThermoFisher Scientific, Massachusetts, USA). qPCR was performed with Apex Probe Master Mix (cat. 42-116P, Genesee, California, USA), TaqMan Gene Expression Probes (Thermofisher Scientific, Massachusetts, USA), and mouse Gapdh (ref. 4352339E, ThermoFisher Scientific, Massachusetts, USA) used as reference. The reactions were run on a BioRad CFX96 Real Time C1000 Touch ThermoCycler and the gene expression fold change was determined via the $\Delta \mathrm{C}_{\mathrm{T}}$ method 62 . The probes we used were designed for the following target genes: Lyve-1 (Mm00475056_m1), Prox-1 (Mm00435969_m1), c-Kit (Mm00445212_m1), Trp53 (Mm01731290_g1), Mdm2 (Mm01233136_m1), Bbc3 (Puma, Mm00519268_m1), and Pmaip1 (Noxa, Mm00451763_m1).

\section{Flow Cytometry}

Embryonic skins of E12.5-E15.5 dpc were harvested using a stereoscope and placed in EHAA media without L-glutamine (Irvine Scientific). Skin was cut into $1 \mathrm{~mm}$ sized pieces and digested for 45 minutes at $37^{\circ} \mathrm{C}$ by $0.25 \mathrm{mg}$ of Liberase $\mathrm{DL}$ (Roche) per $\mathrm{mL}$ of EHAA media and DNAse (Worthington). An equal volume of $0.1 \mathrm{M}$ EDTA in Hank's buffered saline solution without calcium or magnesium was added to the digested cells and incubated for $5 \mathrm{~min}$ at $37^{\circ} \mathrm{C}$. Digested skin was passed through a $100 \mu \mathrm{m}$ strainer and washed with $5 \mathrm{mM}$ EDTA, $2.5 \%$ FBS in EHAA. Stromal cells were stained with CD45 (clone 30F11), PdPn (clone 8.1.1), CD31 (clone 390), and Lyve-1 (clone 223322). Stromal cell subsets were identified by the expression of PdPn and CD31 and the lack of CD45 expression. Blood endothelium populations were classified as CD31 mid or high $\mathrm{PdPn}^{-}$ CD45-. In contrast, lymphatic endothelium cells were categorized as CD31+ PdPn+ CD45-. Cells were run on the DakoCytomation CyAn ADp flow cytometer (Fort Collins, CO) or BD FACS Canto II, acquired using Summit acquisition software and analyzed with FlowJo software (Tree Star, Ashland, OR).

\section{Statistics}

Statistical differences were analyzed using t tests, Chi-Square or one-way ANOVA on GraphPad Prism 8 software. A $P$ value of 0.05 or lower was considered significant.

\section{References}

1. Bursac, S., Brdovcak, M.C., Donati, G. \& Volarevic, S. Activation of the tumor suppressor p53 upon impairment of ribosome biogenesis. Biochim Biophys Acta. 1842, 817-830 (2014).

2. Golomb, L., Volarevic, S. \& Oren, M. p53 and ribosome biogenesis stress: the essentials. Febbs Letters 588, 2571-2579 (2014).

3. Lane, D.P. Cancer. p53, guardian of the genome. Nature 358, 1516 (1992).
4. Vousden, K.H. \& Lu, X. Live or let die: the cell's response to p53. Nature Reviews Cancer 2, 594-604 (2002).

5. Vogelstein, B., Lane, D. \& Levine, A.J. Surfing the p53 network. Nature 408, 307-310 (2000).

6. Parant, J., et al. Rescue of embryonic lethality in Mdm4-null mice by loss of Trp53 suggests a nonoverlapping pathway with MDM2 to regulate p53. Nat Genet 29, 92-95 (2001).

7. Montes de Oca Luna, R., Wagner, D.S. \& Lozano, G. Rescue of early embryonic lethality in mdm2-deficient mice by deletion of p53. Nature 378, 203-206 (1995).

8. Jones, S.N., Roe, A.E., Donehower, L.A. \& Bradley, A. Rescue of embryonic lethality in Mdm2-deficient mice by absence of p53. Nature 378, 206-208 (1995).

9. Mendrysa, S.M., et al. mdm2 Is critical for inhibition of p53 during lymphopoiesis and the response to ionizing irradiation. Mol Cell Biol 23, 462-472 (2003).

10. Terzian, T., et al. Haploinsufficiency of Mdm2 and Mdm4 in tumorigenesis and development. Mol Cell Biol 27, 5479-5485 (2007).

11. Terzian, T. \& Box, N. Genetics of ribosomal proteins: "curiouser and curiouser". PLoS Genet 9, e1003300 (2013).

12. Bowen, M.E. \& Attardi, L.D. The role of p53 in developmental syndromes. J Mol Cell Biol 11, 200-211 (2019).

13. Terzian, T., et al. Rpl27a mutation in the sooty foot ataxia mouse phenocopies high p53 mouse models. J Pathol 224, 540-552 (2011).

14. Alitalo, K., Tammela, T. \& Petrova, T.V. Lymphangiogenesis in development and human disease. Nature 438, 946-953 (2005).

15. Alitalo, K. The lymphatic vasculature in disease. Nat Med 17, 1371-1380 (2011).

16. Gloviczki, M.L. \& Gloviczki, P. Advances and controversies in the contemporary management of chronic lymphedema. Indian Journal of Vascular \& Endovascular Surgery 5, 219-226 (2018).

17. Tammela, T. \& Alitalo, K. Lymphangiogenesis: Molecular Mechanisms and Future Promise. Cell 140, 460-476 (2010).

18. Hong, Y.K., et al. Prox1 is a master control gene in the program specifying lymphatic endothelial cell fate. Dev Dyn 225, 351-357 (2002).

19. Oliver, G. \& Srinivasan, R.S. Endothelial cell plasticity: how to become and remain a lymphatic endothelial cell. Development 137, 363-372 (2010).

20. Yang, Y. \& Oliver, G. Development of the mammalian lymphatic vasculature. Journal of Clinical Investigation 124, 888-897 (2014).

21. Wigle, J.T. \& Oliver, G. Prox1 function is required for the development of the murine lymphatic system. Cell 98, 769-778 (1999).

22. Oliver, G. Lymphatic vasculature development. Nat Rev Immunol 4, 35-45 (2004).

23. Francois, M., et al. Segmental territories along the cardinal veins generate lymph sacs via a ballooning mechanism during embryonic lymphangiogenesis in mice. Dev Biol 364, 89-98 (2012).

24. Yang, Y., et al. Lymphatic endothelial progenitors bud from the cardinal vein and intersomitic vessels in mammalian embryos. Blood 120, 2340-2348 (2012).

25. Hagerling, R., et al. A novel multistep mechanism for initial lymphangiogenesis in mouse embryos based on ultramicroscopy. EMBO J 32, 629-644 (2013).

26. Kazenwadel, J. \& Harvey, N.L. Morphogenesis of the Lymphatic Vasculature: A Focus on New Progenitors and Cellular Mechanism Important for Constructing Lymphatic Vessels. Developmental Dynamics 245, 209-219 (2016).

27. Srinivasan, R.S. \& Oliver, G. Prox1 dosage controls the number of lymphatic endothelial cell progenitors and the formation of the lymphovenous valves. Genes Dev 25, 2187-2197 (2011).

28. Geng, X., et al. Multiple mouse models of primary lymphedema exhibit distinct defects in lymphovenous valve development. Dev Biol 409, 218-233 (2016).

29. Brouillard, P., Boon, L. \& Vikkula, M. Genetics of lymphatic anomalies. J Clin Invest 124, 898-904 (2014).

30. Rockson, S.G., et al. Pilot studies demonstrate the potential benefits of antiinflammatory therapy in human lymphedema. $\mathrm{JCl}$ Insight 3(2018).

31. Tian, W., et al. Leukotriene B4 antagonism ameliorates experimental lymphedema. Sci Transl Med 9(2017). 
bioRxiv preprint doi: https://doi.org/10.1101/2020.03.18.992784; this version posted April 1, 2020. The copyright holder for this preprint (which was not certified by peer review) is the author/funder, who has granted bioRxiv a license to display the preprint in perpetuity. It is made available under aCC-BY-NC-ND 4.0 International license.

32. Sucov, H.M., et al. RXRa mutant mice establish a genetic basis for vitamin A signaling in heart morphogenesis. Genes and Development 8, 1007-1018 (1994).

33. Paszty, C., et al. Lethal a-thalassaemia created by gene targeting in mice and its genetic rescue. Nature Genetics 11, 33-39 (1995)

34. Grier, J.D., Xiong, S., Elizondo-Fraire, A.C., Parant, J.M. \& Lozano, G. Tissue-specific differences of p53 inhibition by Mdm2 and Mdm4. Mol Cell Biol 26, 192-198 (2006).

35. Xiong, S. Mouse models of Mdm2 and Mdm4 and their clinical implications. Chin J Cancer 32, 371-375 (2013).

36. Boesten, L.S., et al. Mdm2, but not Mdm4, protects terminally differentiated smooth muscle cells from p53-mediated caspase-3independent cell death. Cell Death Differ 13, 2089-2098 (2006).

37. Bohmer, R., et al. Regulation of developmental lymphangiogenesis by Syk(+) leukocytes. Dev Cell 18, 437-449 (2010).

38. Stanczuk, L., et al. cKit Lineage Hemogenic Endothelium-Derived Cells Contribute to Mesenteric Lymphatic Vessels. Cell Rep (2015).

39. Shadfan, M., Lopez-Pajares, V. \& Yuan, Z.-M. MDM2 and MDMX Alone and together in regulation of p53. Translational Cancer Research 1, 88-99 (2012).

40. Finlon, J.M., Burchill, M.A. \& Tamburini, B.A. Digestion of the murine liver for flow cytometric analysis of lymphatic endothelial cells. Journal of Visual Experiments 7(2019).

41. Kedl, R.M., et al. Migratory dendritic cells acquire and present lymphatic endothelial cell-archived antigens during lymph node contraction. Nature Communications 8(2017).

42. Tamburini, B.A., Burchill, M.A. \& Kedl, R.M. Antigen capture and archiving by lymphatic endothelial cells following vaccination or viral infection. Nature Communications 5(2014).

43. Tamburini, B.A., et al. PD-1 Blockade During Post-partum Involution Reactivates the Anti-tumor Response and Reduces Lymphatic Vessel Density. Frontiers in Immunology 10(2019).

44. Tamburini, B.A., et al. Chronic Liver Disease in Humans Causes Expansion and Differentiation of Liver Lymphatic Endothelial Cells. Frontiers in Immunology 10(2019).

45. Komarov, P.G., et al. A chemical inhibitor of p53 that protects mice from the side effects of cancer therapy. Science 285, 1733-1737 (1999).

46. Leonova, K.I., et al. A small molecule inhibitor of p53 stimulates amplification of hematopoietic stem cells but does not promote tumor development in mice. Cell Cycle 9, 1434-1443 (2010).

47. Srinivasan, R.S., et al. The Prox1-Vegfr3 feedback loop maintains the identity and the number of lymphatic endothelial cell progenitors. Genes Dev 28, 2175-2187 (2014).

48. Xiong, S., Van Pelt, C.S., Elizondo-Fraire, A.C., FernandezGarcia, B. \& Lozano, G. Loss of Mdm4 results in p53-dependent dilated cardiomyopathy. Circulation 115, 2925-2930 (2007).

49. McGowan, K.A., et al. Ribosomal mutations cause p53-mediated dark skin and pleiotropic effects. Nat Genet 40, 963-970 (2008)

50. Xiong, X., et al. Ribosomal protein S27-like is a physiological regulator of p53 that suppresses genomic instability and tumorigenesis. Elife 3, e02236 (2014)

51. Jones, S.N., Roe, A.E., D'onehower, L.A. \& Bradley, A. Rescue of embryonic lethality in Mdm2-deficient mice by absence of p53. Nature 378, 206-208 (1995).

52. Wlodarski, M.W., et al. Recurring mutations in RPL15 are linked to hydrops fetalis and treatment independence in Diamond-Blackfan anemia. The Hematology Journal 103, 949-958 (2018).

53. Bellini, C., et al. Etiology of non-immune hydrops fetalis: An update. Am J Med Genet A 167A, 1082-1088 (2015).

54. Donehower, L., et al. Mice deficient for p53 are developmentally normal but susceptible to spontaneous tumors. Nature $356,215-$ 221 (1992).

55. Martinez-Corral, I., et al. Nonvenous origin of dermal lymphatic vasculature. Circ Res 116, 1649-1654 (2015)

56. Bernier-Latmani, J., Sabine, A. \& Petrova, T.V. Meet me in the middle: dual origins of dermal lymphatic vasculature in mammals. Circ Res 116, 1630-1632 (2015).

57. Gousopoulos, E., et al. An Important Role of VEGF-C in Promoting Lymphedema Development. J Invest Dermatol 137, 1995-2004 (2017).

58. Stanley G. Rockson, Vaughan Keeley, Sharon Kilbreath, Andrzej Szuba \& Towers, A. Cancer-associated secondary lymphoedema. Primer 5, 1-16 (2019).
59. Jacks, T., et al. Tumor spectrum analysis in p53-mutant mice. Current Biology 4, 1-7 (1994).

60. Deng, J.M., et al. Generation of Viable Male and Female Mice from Two Fathers. Biology of Reproduction 84, 613-618 (2011)

61. Dierkes, C., Scherzinger, A. \& Kiefer, F. Three-Dimensional Visualization of the Lymphatic Vasculature. Methods Mol Biol 1846, 1-18 (2018).

62. Livak, K.J. \& Schmittgen, T.D. Analysis of relative gene expression data using real-time quantitative PCR and the 2-delta delta CT Method. Methods 25, 402-408 (2001). 